Research Paper

\title{
Biomarkers for Predicting Malignant Pleural Mesothelioma in a Mexican Population
}

\author{
Guadalupe Aguilar-Madrid ${ }^{1 * \bowtie}$, Beate Pesch ${ }^{2 *}$, Emma S Calderón-Aranda ${ }^{3}$, Katarzyna Burek ${ }^{2}$, Carmina
} Jiménez-Ramírez ${ }^{1,4}$, Cuauhtémoc Arturo Juárez-Pérez ${ }^{1}$, María Dolores Ochoa-Vázquez ${ }^{5}$, Luis Torre-Bouscoulet ${ }^{6}$, Leonor Concepción Acosta-Saavedra ${ }^{3}$, Isabel Sada-Ovalle7, Jorge García-Figueroa7, Isabel Alvarado-Cabrero ${ }^{8}$, Patricia Castillo-González ${ }^{9}$, Alejandra Renata Báez-Saldaña9 ${ }^{9}$, José Rogelio Pérez-Padilla ${ }^{10}$, Juvencio Osnaya-Juárez5 , Rosa María Rivera-Rosales"11, Eric Marco García-Bazán12, Yolanda Lizbeth Bautista-Aragón ${ }^{13}$, Elimelec Lazcano-Hernandez ${ }^{12}$, Daniel Alejandro Munguía-Canales'14, Luis Marcelo Argote-Greene'5, Dirk Taeger², Daniel Gilbert Weber², Swaantje Casjens², Irina Raiko², Thomas Brüning², Georg Johnen² ${ }^{\bowtie}$

1. Research Unit Health at Work, XXI Century National Medical Center (CMNSXXI), Instituto Mexicano del Seguro Social (IMSS), Mexico City, Mexico

2. Institute for Prevention and Occupational Medicine of the German Social Accident Insurance, Institute of the Ruhr-University Bochum (IPA), Bochum, Germany

3. Department of Toxicology, Center for Research and Advanced Studies, CINVESTAV, Mexico City, Mexico

4. Clinical Analysis Laboratory, Traumatology Hospital "Dr. Victorio De la Fuente Narvaez", IMSS, Mexico City, Mexico

5. Pneumology Service of the General Hospital, Medical Center La Raza, IMSS, Mexico City, Mexico

6. Clinical Research, National Institute of Respiratory Diseases (INER), Mexico City, Mexico

Integrative Immunology Laboratory, INER, Mexico City, Mexico

8. Service Pathology, High Specialty Medical Unit (UMAE), Oncology Hospital, CMNSXXI, IMSS, Mexico City, Mexico

9. Clinical Oncology Pneumology Service, INER, Mexico City, Mexico

10. Research Department, INER, Mexico City, Mexico

1. Department of Pathology, INER, Mexico City, Mexico

12. Thorax Service, Oncology Hospital, High Specialty Medical Unit (UMAE), CMNSXXI, IMSS, Mexico City, Mexico

13. Medical Oncology, Oncology Hospital, High Specialty Medical Unit (UMAE), CMNSXXI, IMSS, Mexico City, Mexico

14. Service Chest Surgery, Hospital Cardiology, CMNSXXI, IMSS, Mexico City, Mexico

15. Thoracic Surgery, Case Western Reserve University Hospitals of Cleveland, USA

* These authors contributed equally to this work

$\triangle$ Corresponding authors: Georg Johnen, PhD, Institute for Prevention and Occupational Medicine of the German Social Accident Insurance, Institute of the Ruhr-University Bochum (IPA), Bürkle-de-la-Camp-Platz 1, 44789 Bochum, Germany, Tel.: +49 2343024509 , Fax: +49 234 3024505 , e-mail: johnen@ipa-dguv.de and Guadalupe Aguilar-Madrid, PhD, Unidad de Investigación en Salud en el Trabajo, Instituto Mexicano del Seguro Social, Centro Médico Nacional Siglo XXI, Av. Cuauhtémoc No. 330, CP 06720, Ciudad de México, México, Tel.: +52 555761 0725, e-mail: gpeaguilarm@gmail.com

(C) Ivyspring International Publisher. This is an open access article distributed under the terms of the Creative Commons Attribution (CC BY) license (https://creativecommons.org/licenses/by/4.0/). See http://ivyspring.com/terms for full terms and conditions.

Received: 2017.11.20; Accepted: 2018.04.09; Published: 2018.06.04

\begin{abstract}
Background: Diagnosis of malignant pleural mesothelioma (MPM) remains a challenge, especially when resources in pathology are limited. The study aimed to evaluate cost-effective tumor markers to predict the probability of MPM in plasma samples in order to accelerate the diagnostic workup of the tissue of potential cases.

Methods: We conducted a case-control study stratified by gender, which included 75 incident cases with MPM from three Mexican hospitals and 240 controls frequency-matched by age and year of blood drawing. Plasma samples were obtained to determine mesothelin, calretinin, and thrombomodulin using enzyme-linked immunosorbent assays (ELISAs). We estimated the performance of the markers based on the area under the curve (AUC) and predicted the probability of an MPM diagnosis of a potential case based on the marker concentrations.

Results: Mesothelin and calretinin, but not thrombomodulin were significant predictors of a diagnosis of MPM with AUCs of 0.90 (95\% Cl: $0.85-0.95), 0.88$ (95\% Cl: $0.82-0.94)$, and 0.51 (95\% Cl: $0.41-0.61)$ in males, respectively. For MPM diagnosis in men we estimated a true positive rate of 0.79 and a false positive rate of 0.11 for mesothelin. The corresponding figures for calretinin were 0.81 and 0.18 , and for both markers combined 0.84 and 0.11 , respectively.

Conclusions: We developed prediction models based on plasma concentrations of mesothelin and calretinin to estimate the probability of an MPM diagnosis. Both markers showed a good performance and could be used to accelerate the diagnostic workup of tissue samples in Mexico.
\end{abstract}

Key words: mesothelioma, mesothelin, calretinin, diagnostic marker, asbestos. 


\section{Introduction}

Malignant pleural mesothelioma (MPM) is an extremely lethal cancer strongly associated with exposure to asbestos. Asbestos is still an important commodity in global trade [1]. About 50 countries banned the use of asbestos, but not yet Mexico, Colombia, Brazil, and many other countries worldwide. Even after cessation of exposure, the risk of developing an MPM is strongly elevated because of the long latency of this cancer [2]. Efforts are under way to build international research networks in asbestos-related disease prevention [3]. The Project 'MoMar' (Molecular Markers), for example, aims to identify and validate minimally-invasive tumor markers for the early detection of MPM with study groups from Mexico, Greece, Australia, and Germany $[4,5]$.

MPM diagnosis remains a challenge, and the prognosis is poor [6]. In combination with imaging methods, tumor markers have been suggested to improve the diagnostic workup and to enhance survival $[7,8]$. Three markers have been selected in this study to evaluate their potential to assist the diagnostic workup. Mesothelin has been the most promising blood-based tumor marker so far $[9,10]$ and the well-established immunohistochemical marker calretinin was shown to be elevated in plasma samples of MPM patients [5, 11]. Thrombomodulin, another immunohistochemical marker for MPM [12], was reasoned to be also a possible candidate for blood-based MPM detection. The involvement of these proteins in key processes of cancer development, such as proliferation and angiogenesis, render them also informative for therapeutic targets to improve the so far poor prognosis [13-15].

The Lancet Oncology Commission identified several obstacles to providing optimum cancer services in Latin America and the Caribbean [16]. These limitations include insufficient activities for primary prevention, for example, the ban of asbestos. An update of this comprehensive evaluation addressed remaining challenges such as needs for a higher quality of the histopathological assessments [17]. Due to the limited histopathological capacity, the diagnostic workup of tissue samples from potential cases with MPM can be strongly delayed in Mexico. Blood-based tumor markers that are fast, cost-efficient, and easy to determine may speed-up this process. Thus, the aim of our study was to predict the probability of a diagnosis of MPM based on the plasma levels of mesothelin, calretinin, and thrombomodulin in order to expedite the cases with the most likely MPM diagnosis to a histopathological examination of their tissue samples. Another - more long-term - goal is to find candidate markers for validation in prospective studies. Once validated these markers could be used for the early detection of MPM in screening programs in the future.

\section{Materials and Methods}

\section{Study Population}

A case-control study was conducted comparing tumor marker concentrations in blood samples from 75 incident MPM cases and 240 controls, which were enrolled with participation rates of $98 \%$ and $95 \%$, respectively, in the Valley of Mexico from January 2012 to April 2015. All participants originated from and lived in urban areas. None of the participants were of indigenous origin but $96.4 \%$ were of Mestizo Mexican descent, while the remaining $3.6 \%$ had a more recent European or U.S. American background (first or second generation). Incident cases were recruited from the outpatient and inpatient services of three hospitals, who sought medical care with clinical suspicion of MPM. MPM diagnosis was confirmed by medical oncologists based on clinical examination, imaging tests (X-ray and chest computed tomography), biopsy, and immunohistochemistry. The panel of immunohistochemical biomarkers consisted of calretinin, cytokeratins (CK5/6), Wilms tumor protein (WT-1), vimentin, carcinoembryonic antigen (CEA), and thyroid transcription factor 1 (TTF-1/NKX2-1) [18]. The subtype of MPM was classified according to WHO [19].

Cases were recruited among patients with health insurance at two hospitals from the Mexican Social Security Institute (IMSS) and among uninsured patients from Mexico's National Institute of Respiratory Diseases (INER), a hospital of Mexico's Ministry of Health. All hospitals are referral hospitals for respiratory diseases and associated cancers. Male controls $(n=172)$ were matched by age and year of blood drawing to 63 cases. Female controls $(n=68)$ were also matched by age and year of blood drawing to 12 women with epithelioid MPM at a higher ratio to improve the statistical power. Controls were selected from the National System of Beneficiaries database (SINDO) and from the IMSS' Information System on Severance Pensioners, Advanced and Old Age Workers [20]. The controls were randomly selected from the respective database and invited by phone to voluntarily participate in the study. In addition, controls for INER cases were selected from facilities of the National Institute of Older People (INAPAM), which included day residences, comprehensive care centers, cultural centers, and clubs for elderly people. 
An in-person interview and blood sampling were performed prior to the histopathological confirmation of the diagnosis of MPM. A questionnaire was applied by trained interviewers to assess socio-demographic information, a detailed occupational history, exposure to asbestos, smoking habits, medical history, and other data. Lifetime occupational exposure to asbestos was categorized as ever or never according to a previously published assessment [21]. In brief, an industrial hygienist, who was unaware of the case-control status, estimated the exposure to asbestos according to the worker's job history and a list with information on industries importing asbestos or companies that manufactured asbestos fibers in various forms [22] along with recognized occupational activities and jobs with exposure to asbestos [23-28].

The research protocol was approved by IMSS' National Commission for Scientific Research and Ethics with registration number R-2011-785-069, and by INER's Committee on Science and Bioethics in Research with registration number $\mathrm{C} 30-12$. Prior to inclusion in the study, participants signed a letter of informed consent.

\section{Measurement of Tumor Markers in Plasma}

Blood samples were drawn in three $6.0 \mathrm{ml}$ tubes (EDTA vacutainers) and centrifuged at 2,250 $\mathrm{g}$ for 10 minutes in a laminar flow hood. Plasma and buffy coat were separated and stored at $-80^{\circ} \mathrm{C}$ until analysis. Mesothelin and thrombomodulin ELISAs were performed at CINVESTAV in Mexico City, Mexico. For mesothelin determination, sandwich ELISAs were performed according to the manufacturer's instructions (DY3265, R\&D Systems, Minneapolis, $\mathrm{MN})$. Thrombomodulin concentration was determined according to the manufacturer's instructions (DY3947, R\&D Systems).

Plasma samples were shipped to Germany under stringent frozen conditions and calretinin determination was performed utilizing a sandwich ELISA according to Raiko et al. [11] at the IPA in Bochum, Germany.

\section{Statistical Analysis}

The distributions of the biomarker concentrations were presented by median and interquartile range (IQR). A relatively large number of calretinin concentrations were below the limit of detection (LOD). Therefore, affected percentiles were marked as being less $(<)$ than the respective LOD (Table 1). For the calculations, we set values below LOD to two-thirds of LOD (2/3*LOD). Mesothelin and thrombomodulin concentrations between cases and controls were compared using the Wilcoxon rank-sum test. Because $72 \%$ of calretinin measurements were below LOD, the Peto-Prentice test was applied to compare cases and controls [29, 30].

Odds ratios (ORs) and 95\% confidence intervals (CI) were calculated using unconditional logistic regression models to estimate the relative risk of a diagnosis of MPM based on the log-transformed concentrations of the tumor markers. These models were also used for estimating receiver operating characteristic (ROC) curves and the area under the curve (AUC) for the markers' sensitivity for varying values of specificity. Based on the performance of the markers we predicted the individual probability of a diagnosis of MPM for a male or female subject with the corresponding estimates of the true positive rate (TPR) and false positive rate (FPR) by these logistic regression models. Statistical analyses were performed using SAS software, version 9.4 (SAS Institute Inc., Cary, NC).

\section{Results}

Table 1 depicts the characteristics of the cases and controls. Median age of MPM diagnosis was 64 years for males and 62 years for females. More women than men had never smoked (women: $66.7 \%$ in cases, $64.7 \%$ in controls; men: $33.3 \%$ in cases, $37.8 \%$ in controls). A large fraction of men was classified as ever exposed to asbestos at the workplace $(93.7 \%$ in cases, $70.3 \%$ in controls). The corresponding figures in women were $33.3 \%$ and $17.7 \%$, respectively. Epithelioid MPM was diagnosed for all twelve female cases and for $52(82.5 \%)$ male cases.

Table 2 shows the distribution of the marker concentrations by case-control status in men and women. The concentrations of mesothelin and calretinin but not of thrombomodulin were statistically significant higher in cases than in controls, with distinct IQRs. In men, mesothelin median concentrations were $2.21 \mathrm{nmol} / 1$ in cases and 0.58 $\mathrm{nmol} / \mathrm{l}$ in controls $(\mathrm{p}<0.0001)$. Similar concentrations were determined in women $(2.00 \mathrm{nmol} / 1$ and 0.55 nmol/l, respectively, $\mathrm{p}<0.0001)$. The same pattern was observed for calretinin (men: $0.93 \mathrm{ng} / \mathrm{ml}$ in cases, $<0.07 \mathrm{ng} / \mathrm{ml}$ in controls, $\mathrm{p}<0.0001$; women: 0.86 $\mathrm{ng} / \mathrm{ml}$ in cases, $<0.22 \mathrm{ng} / \mathrm{ml}$ in controls, $\mathrm{p}<0.0037$ ). No differences in thrombomodulin concentrations were observed between cases and controls (men: 2.57 vs. $2.30 \mathrm{ng} / \mathrm{ml}, \mathrm{p}=0.81$; women: 2.25 vs. $2.05 \mathrm{ng} / \mathrm{ml}$, $p=0.84$ ). In addition, we show the distribution of the concentrations in subgroups stratified by age and exposure to asbestos. 
Table 1. Description of the study population of cases with MPM and controls from Mexico

\begin{tabular}{|c|c|c|c|c|c|c|}
\hline & Male & & & Female & & \\
\hline Characteristics & Cases N (\%) & Controls N (\%) & $P$-value & Cases N (\%) & Controls N (\%) & $P$-value \\
\hline Total & 63 & 172 & & 12 & 68 & \\
\hline \multicolumn{7}{|l|}{ Age (years) } \\
\hline Median (IQR) & $64(56-72)$ & $62(55-71)$ & 0.59 & $62(53-68)$ & $61(53-70)$ & 0.82 \\
\hline \multicolumn{7}{|l|}{ Histologic diagnosis } \\
\hline Epithelioid & $52(82.5)$ & & & $12(100)$ & & \\
\hline Sarcomatoid & $3(4.8)$ & & & - & & \\
\hline Other & 7 (11.1) & & & - & & \\
\hline Not specified & $1(1.6)$ & & & - & & \\
\hline Smoking status & & & 0.0003 & & & - \\
\hline Never smoker & $21(33.3)$ & $65(37.8)$ & & $8(66.7)$ & $44(64.7)$ & \\
\hline Former/current smoker & $42(66.7)$ & $107(62.2)$ & & $4(33.3)$ & $24(35.3)$ & \\
\hline Occupational exposure to asbestos & & & $<0.0001$ & & & 0.24 \\
\hline Never & $4(6.3)$ & $51(29.7)$ & & $8(66.7)$ & $56(82.4)$ & \\
\hline Ever & $59(93.7)$ & $121(70.3)$ & & $4(33.3)$ & $12(17.7)$ & \\
\hline
\end{tabular}

aContinuous variables were compared using the Wilcoxon rank-sum test, categorical variables were compared using Fisher's exact test. IQR: interquartile range

Table 2. Distribution of biomarker concentrations in plasma samples from cases with MPM and controls from Mexico

\begin{tabular}{|c|c|c|c|c|c|c|c|c|}
\hline & & \multicolumn{3}{|c|}{ Cases } & \multicolumn{4}{|c|}{ Controls } \\
\hline & & $\mathrm{N}$ & Median & IQR & $\mathrm{N}$ & Median & IQR & $P$-value \\
\hline \multicolumn{9}{|c|}{ Mesothelin $(\mathrm{nmol} / \mathrm{L})$} \\
\hline Males & & 63 & 2.21 & $1.37-3.93$ & 170 & 0.58 & $0.40-0.87$ & $<0.0001$ \\
\hline \multirow[t]{2}{*}{ Age } & $<70$ years & 41 & 2.04 & $1.28-3.77$ & 120 & 0.52 & $0.38-0.76$ & \\
\hline & $\geq 70$ years & 22 & 2.75 & $2.14-3.93$ & 50 & 0.76 & $0.48-1.02$ & \\
\hline \multicolumn{9}{|c|}{ Occupational exposure to asbestos } \\
\hline & Never & 4 & 1.10 & $0.66-2.76$ & 51 & 0.61 & $0.43-0.85$ & \\
\hline & Ever & 59 & 2.29 & $1.56-3.93$ & 119 & 0.55 & $0.40-0.89$ & \\
\hline \multicolumn{2}{|c|}{ Females } & 12 & 2.00 & $1.31-5.40$ & 65 & 0.55 & $0.41-0.79$ & $<0.0001$ \\
\hline \multirow[t]{2}{*}{ Age } & $<70$ years & 9 & 1.46 & $1.28-3.24$ & 48 & 0.55 & $0.41-0.78$ & \\
\hline & $\geq 70$ years & 3 & 6.20 & $2.13-9.90$ & 17 & 0.59 & $0.31-0.95$ & \\
\hline \multicolumn{9}{|c|}{ Occupational exposure to asbestos } \\
\hline & Never & 8 & 1.80 & $1.29-5.40$ & 53 & 0.55 & $0.38-0.79$ & \\
\hline & Ever & 4 & 2.56 & $1.57-5.01$ & 12 & 0.55 & $0.45-0.87$ & \\
\hline \multicolumn{9}{|c|}{ Calretinin $(n g / m L)$} \\
\hline \multicolumn{2}{|l|}{ Males } & 62 & 0.93 & $<0.45-2.18$ & 172 & $<0.07$ & $<0.01-<0.22$ & $<0.0001$ \\
\hline \multirow[t]{2}{*}{ Age } & $<70$ years & 40 & 1.00 & $<0.31-2.50$ & 120 & $<0.08$ & $<0.01-<0.23$ & \\
\hline & $\geq 70$ years & 22 & 0.85 & $0.58-1.98$ & 52 & $<0.06$ & $<0.01-<0.22$ & \\
\hline \multicolumn{9}{|c|}{ Occupational exposure to asbestos } \\
\hline & Never & 4 & 1.41 & $<0.74-2.16$ & 51 & $<0.06$ & $<0.01-<0.20$ & \\
\hline & Ever & 58 & 0.86 & $<0.45-2.18$ & 121 & $<0.08$ & $<0.01-<0.24$ & \\
\hline Femal & & 12 & 0.86 & $<0.25-1.33$ & 68 & $<0.22$ & $<0.10-0.48$ & 0.0037 \\
\hline \multirow[t]{2}{*}{ Age } & $<70$ years & 9 & $<0.35$ & $<0.24-1.12$ & 50 & $<0.22$ & $<0.12-0.48$ & \\
\hline & $\geq 70$ years & 3 & 3.09 & $0.97-4.98$ & 18 & $<0.18$ & $<0.03-<0.46$ & \\
\hline \multicolumn{9}{|c|}{ Occupational exposure to asbestos } \\
\hline & Never & 8 & 1.04 & $<0.55-2.16$ & 56 & $<0.22$ & $<0.10-0.48$ & \\
\hline & Ever & 4 & $<0.25$ & $<0.13-<0.85$ & 12 & $<0.27$ & $<0.08-<0.47$ & \\
\hline \multicolumn{9}{|c|}{ Thrombomodulin $(n g / m L)$} \\
\hline \multicolumn{2}{|c|}{ Males } & 54 & 2.57 & $1.71-3.18$ & 124 & 2.30 & $1.84-2.85$ & 0.81 \\
\hline \multirow[t]{2}{*}{ Age } & $<70$ years & 35 & 2.01 & $1.66-2.90$ & 96 & 2.24 & $1.79-2.76$ & \\
\hline & $\geq 70$ years & 19 & 3.04 & $1.97-3.41$ & 28 & 2.79 & $2.19-3.38$ & \\
\hline \multicolumn{9}{|c|}{ Occupational exposure to asbestos } \\
\hline & Never & 3 & 2.84 & $1.72-3.17$ & 29 & 2.22 & $1.70-2.87$ & \\
\hline & Ever & 51 & 2.53 & $1.67-3.25$ & 95 & 2.31 & $1.86-2.83$ & \\
\hline \multicolumn{2}{|c|}{ Females } & 10 & 2.25 & $1.77-2.43$ & 63 & 2.05 & $1.62-2.72$ & 0.84 \\
\hline \multirow[t]{2}{*}{ Age } & $<70$ years & 8 & 2.07 & $1.59-2.36$ & 47 & 2.01 & $1.58-2.51$ & \\
\hline & $\geq 70$ years & 2 & 2.50 & $2.27-2.73$ & 16 & 2.26 & $2.06-3.14$ & \\
\hline \multicolumn{9}{|c|}{ Occupational exposure to asbestos } \\
\hline & Never & 6 & 2.10 & $1.77-2.30$ & 51 & 2.05 & $1.66-2.72$ & \\
\hline & Ever & 4 & 2.33 & $1.82-3.19$ & 12 & 2.09 & $1.26-2.66$ & \\
\hline
\end{tabular}

aMesothelin and thrombomodulin concentrations were compared using the Wilcoxon rank-sum test, calretinin concentrations were compared using the Peto-Prentice test. IQR: interquartile range; '<' indicates percentiles below the limit of detection (LOD) 
Table 3 presents the ORs with 95\% CI as estimates of the relative risk of an MPM per unit increase of the log-transformed concentrations of the markers. Whereas thrombomodulin was not associated with the development of MPM in men $(\mathrm{OR}=1.05,95 \% \mathrm{CI}$ 0.44-2.48) and women $(\mathrm{OR}=1.02$, 95\% CI 0.19-5.47), both mesothelin and calretinin were significant factors. The MPM risks per unit increase of the mesothelin and calretinin concentrations were 14.51 (95\% CI 6.96-30.28) and 3.03 (95\% CI 2.20-4.18) in men and 28.57 (95\% CI 4.09-199.4) and 2.69 (95\% CI 1.31-5.56) in women, respectively. In the multivariate analysis mesothelin appears to be more clearly associated with MPM risk than calretinin for males and females.
Using ROC analyses, the calculated AUCs for the mesothelin concentrations were $0.90 \quad(95 \% \quad \mathrm{CI}$ $0.85-0.95)$ in men and 0.92 (95\% CI 0.79-1.00) in women. The AUCs for calretinin were slightly weaker, with 0.88 (95\% CI 0.82-0.94) in men and 0.77 (95\% CI 0.61-0.93) in women (Figure 1). Due to the low AUC, thrombomodulin was excluded from further analysis. Based on the plasma concentrations of mesothelin and calretinin we predicted the individual probability of an MPM in a male or female subject using the prediction formulas presented in Tables 4 and 5, respectively, resulting in estimated TPRs and FPRs for the whole study population stratified to probabilities ranging from $10 \%$ to $90 \%$.

Table 3. Odds ratios from logistic regression analyses as estimates of the relative risk for an MPM based on the plasma concentrations in cases and controls

\begin{tabular}{|c|c|c|c|c|}
\hline Study group & Characteristics & Intercept & Coefficient & OR $(95 \% \mathrm{CI})$ \\
\hline \multirow[t]{8}{*}{ Males } & Models & & & \\
\hline & Univariate & & & \\
\hline & $\ln ($ Mesothelin) $[\mathrm{nmol} / \mathrm{L}]$ & -1.15 & 2.68 & $14.51(6.96-30.28)$ \\
\hline & $\ln ($ Calretinin $)[\mathrm{ng} / \mathrm{mL}]$ & 0.55 & 1.11 & $3.03(2.20-4.18)$ \\
\hline & $\ln$ (Thrombomodulin) $[\mathrm{ng} / \mathrm{mL}]$ & -0.87 & 0.04 & $1.05(0.44-2.48)$ \\
\hline & Multivariate & & & \\
\hline & $\ln ($ Mesothelin) $[\mathrm{nmol} / \mathrm{L}]$ & -0.17 & 2.11 & $8.26(3.77-18.10)$ \\
\hline & $\ln ($ Calretinin $)[\mathrm{ng} / \mathrm{mL}]$ & & 0.59 & $1.80(1.31-2.47)$ \\
\hline \multirow[t]{8}{*}{ Females } & Models & & & \\
\hline & Univariate & & & \\
\hline & $\ln ($ Mesothelin) $[\mathrm{nmol} / \mathrm{L}]$ & -1.77 & 3.35 & $28.57(4.09-199.4)$ \\
\hline & $\ln ($ Calretinin) $[\mathrm{ng} / \mathrm{mL}]$ & -0.72 & 0.99 & $2.69(1.31-5.56)$ \\
\hline & $\ln ($ Thrombomodulin) $[\mathrm{ng} / \mathrm{mL}]$ & -1.86 & 0.02 & $1.02(0.19-5.47)$ \\
\hline & Multivariate & & & \\
\hline & $\ln ($ Mesothelin) $[\mathrm{nmol} / \mathrm{L}]$ & -1.36 & 3.08 & $21.86(3.13-152.51)$ \\
\hline & $\ln ($ Calretinin $)[\mathrm{ng} / \mathrm{mL}]$ & & 0.33 & $1.39(0.67-2.88)$ \\
\hline
\end{tabular}

OR: odds ratio; CI: confidence interval; ln: natural logarithm

A

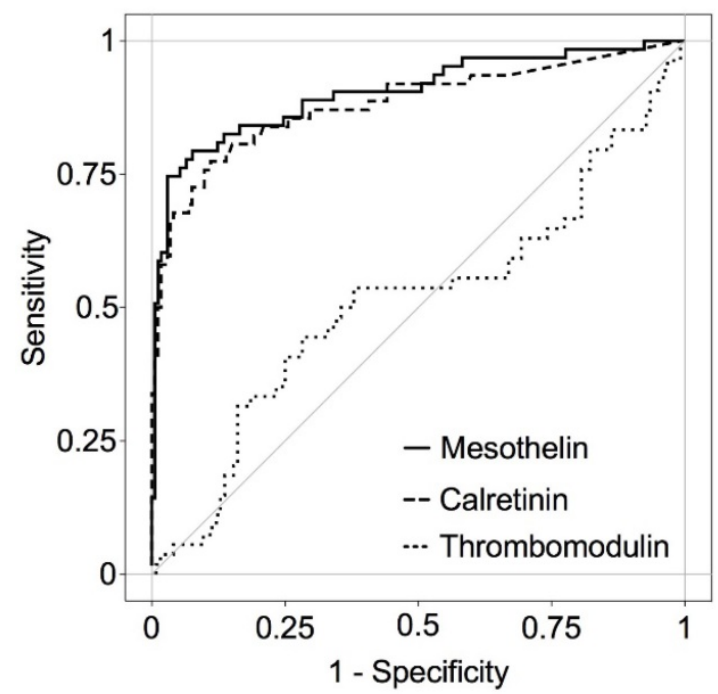

B

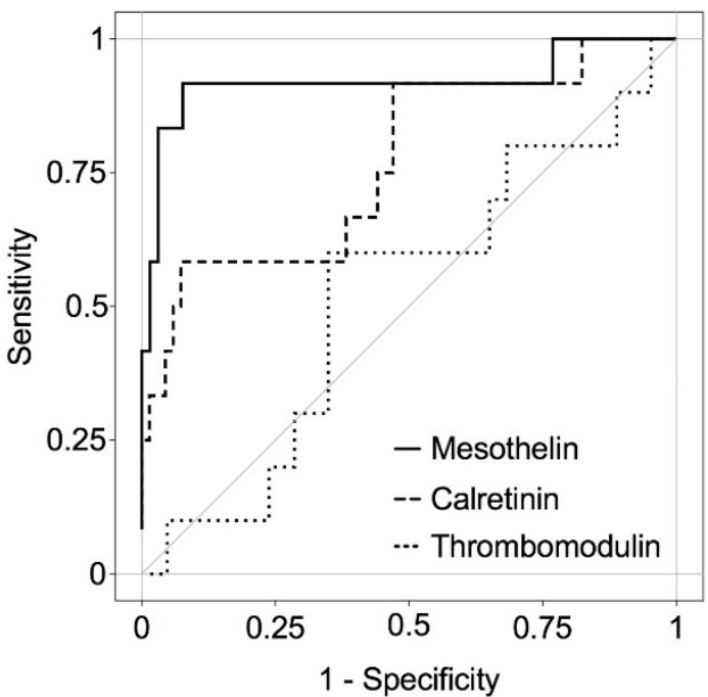

Figure 1. ROC curves of MPM biomarkers in incident cases and controls by gender (all Cls were $95 \%)$. A, Males: mesothelin (AUC $=0.90, \mathrm{Cl}: 0.85-0.95)$, calretinin (AUC $=0.88$, $\mathrm{Cl}: 0.82-0.94)$, and thrombomodulin (AUC=0.51, Cl:0.41-0.61) B, Females: mesothelin (AUC=0.92, Cl:0.79-1.00), calretinin (AUC=0.77, Cl:0.61-0.93), and thrombomodulin $(\mathrm{AUC}=0.52, \mathrm{Cl}: 0.32-0.72)$ 
Table 4. Performance of mesothelin and calretinin in men and the probability of a diagnosis of MPM, conditional on the observed biomarker concentrations

\begin{tabular}{|c|c|c|c|c|c|c|c|c|c|c|}
\hline \multirow{3}{*}{$\begin{array}{l}\text { Probability } \\
\%\end{array}$} & \multicolumn{10}{|l|}{ Males } \\
\hline & \multicolumn{3}{|l|}{ Model 1} & \multicolumn{3}{|l|}{ Model 2} & \multicolumn{4}{|l|}{ Model 3} \\
\hline & Cut off Mesothelin [nmol/L] & TPR & FPR & Cut off Calretinin $[\mathrm{ng} / \mathrm{mL}]$ & TPR & FPR & Cut off Mesothelin [nmol/L] & Cut off Calretinin [ng/mL] & TPR & FPR \\
\hline 90 & 3.48 & 0.33 & 0.01 & 4.47 & 0.10 & 0 & 3.66 & 0.85 & 0.37 & 0.01 \\
\hline 80 & 2.62 & 0.43 & 0.01 & 2.18 & 0.26 & 0 & 2.15 & 1 & 0.53 & 0.01 \\
\hline 70 & 2.09 & 0.57 & 0.01 & 1.36 & 0.40 & 0.01 & 1.08 & 4.25 & 0.63 & 0.01 \\
\hline 60 & 1.81 & 0.63 & 0.03 & 0.87 & 0.52 & 0.02 & 1.46 & 0.79 & 0.73 & 0.03 \\
\hline 50 & 1.56 & 0.73 & 0.03 & 0.62 & 0.68 & 0.05 & 0.74 & 3.82 & 0.76 & 0.05 \\
\hline 40 & 1.31 & 0.78 & 0.06 & 0.41 & 0.76 & 0.10 & 0.97 & 0.74 & 0.84 & 0.07 \\
\hline 30 & 1.12 & 0.79 & 0.11 & 0.29 & 0.81 & 0.18 & 1.01 & 0.33 & 0.84 & 0.11 \\
\hline 20 & 0.92 & 0.84 & 0.22 & 0.18 & 0.87 & 0.34 & 0.65 & 0.58 & 0.85 & 0.14 \\
\hline 10 & 0.68 & 0.90 & 0.40 & 0.09 & 0.92 & 0.49 & 1.31 & 0.01 & 0.92 & 0.33 \\
\hline
\end{tabular}

Probability was used to estimate TPR and FPR: Probability $=1 /\left(1+\mathrm{e}^{\wedge}(-\varphi)\right)$. Logistic regression models: $(1)$ with log-mesothelin as predictor, $\varphi=\exp [-1.15+2.68$ * $\ln ($ mesothelin $)] ;$ (2) with log-calretinin as predictor, $\varphi=\exp [0.55+1.11 * \ln ($ calretinin $)] ;(3)$ with $\log$-mesothelin and $\log$-calretinin, $\varphi=\exp [-0.17+2.11 * \ln ($ mesothelin $)+0.59 *$ $\ln ($ calretinin)]. TPR: true positive rate; FPR: false positive rate

Table 5. Performance of mesothelin and calretinin in women and the probability of a diagnosis of MPM, conditional on the observed biomarker concentrations

\begin{tabular}{|c|c|c|c|c|c|c|c|c|c|c|}
\hline \multirow{3}{*}{$\begin{array}{l}\text { Probability } \\
\%\end{array}$} & \multicolumn{10}{|l|}{ Females } \\
\hline & \multicolumn{3}{|l|}{ Model 1} & \multicolumn{3}{|l|}{ Model 2} & \multicolumn{4}{|l|}{ Model 3} \\
\hline & Cut off Mesothelin [nmol/L] & TPR & FPR & Cut off Calretinin $[\mathrm{ng} / \mathrm{mL}]$ & TPR & FPR & Cut off Mesothelin [nmol/L] & Cut off Calretinin [ng/mL] & TPR & FPR \\
\hline 90 & 3.24 & 0.42 & 0 & - & 0 & 0 & - & - & 0.33 & 0 \\
\hline 80 & - & 0.42 & 0 & - & 0 & 0 & 2.13 & 3.09 & 0.50 & 0 \\
\hline 70 & - & 0.42 & 0.02 & 4.98 & 0.08 & 0 & - & - & 0.50 & 0.02 \\
\hline 60 & 1.87 & 0.58 & 0.02 & 3.09 & 0.17 & 0 & - & - & 0.58 & 0.02 \\
\hline 50 & - & 0.58 & 0.02 & - & 0.17 & 0 & - & - & 0.58 & 0.02 \\
\hline 40 & 1.48 & 0.58 & 0.03 & 1.44 & 0.25 & 0 & 1.35 & 1.23 & 0.75 & 0.02 \\
\hline 30 & 1.35 & 0.75 & 0.03 & 0.97 & 0.50 & 0.06 & 1.21 & 1.12 & 0.75 & 0.05 \\
\hline 20 & 1.14 & 0.92 & 0.12 & 0.49 & 0.58 & 0.24 & 1.05 & 0.72 & 0.83 & 0.09 \\
\hline 10 & 0.90 & 0.92 & 0.20 & 0.23 & 0.92 & 0.49 & 0.90 & 0.23 & 0.92 & 0.20 \\
\hline
\end{tabular}

Utilizing mesothelin alone a pre-defined 30\% probability for the diagnosis of MPM in an individual was associated with high TPRs in the study population ( 0.79 in men and 0.75 in women) and moderate FPRs (0.11 in men and 0.03 in women). For comparison, a high individual probability of $80 \%$ was associated with a moderate TPR of 0.43 and a low FPR of 0.01 in men and similar rates in women. An increase of the TPR, with stable FPR, could be achieved in men by including calretinin into the decision model (30\% probability: TPR $=0.84,80 \%$ probability: TPR $=0.53$ ). Both markers are moderately correlated in plasma samples of cases (Kendall's tau correlation coefficient: $0.37, \mathrm{p}<0.0001$ ). The cut off values in Tables 4 and 5 can be used to apply the biomarkers to different tasks/issues, e.g., screening or optimizing histopathological diagnostic workup.

\section{Discussion}

MPM is an aggressive cancer and difficult to diagnose, particularly at early stages. Reliable tumor markers could improve the diagnosis of MPM, including early stages $[11,31,32]$. In some countries like Mexico, the resources for histopathological diagnosis of suspected cases are limited. MPM is also a rare disease, with a very low probability of an individual suffering from this type of cancer. Additional information on the blood concentrations of tumor markers can enhance an individual's probability. Here, we provided a prediction model for the probability of a diagnosis of MPM ranging from $10 \%$ to $90 \%$ in order to optimize the histopathological diagnostic workup of tissue samples.

Formulas given in Tables 4 and 5 can be used to calculate the probability of a subject for a diagnosis of MPM by gender for given plasma concentrations of mesothelin and calretinin. A high individual probability of a subject such as $90 \%$ is associated with a high tumor marker level. This high tumor marker concentration implies that the rates of true positives and of false positives at group level are lower. By contrast, a low individual probability of a subject such as $10 \%$ can be achieved at a lower tumor marker level. Hence, the corresponding rates of true positives and of false positives are higher.

For men, we observed a good individual performance of mesothelin as well as calretinin. A combination of both markers showed moderate improvement of the performance. For screening, one would want to achieve a high probability in order to keep the FPR low. For example, at $80 \%$ probability for 
a diagnosis of MPM mesothelin and calretinin showed a low rate of $1 \%$ and $0 \%$ false-positive decisions corresponding to $43 \%$ and $26 \%$ true-positive decisions, respectively. The combination of both markers resulted in 1\% false-positive and 53\% true-positive decisions. However, for diagnostic workup a lower probability can be accepted. Using a probability of $30 \%$, mesothelin and calretinin showed $11 \%$ and $18 \%$ false-positive decisions with corresponding $79 \%$ and $81 \%$ true-positive decisions, respectively. The combination of both markers improved the performance to an acceptable $11 \%$ false-positive decisions and a relatively high rate of $84 \%$ true-positive decisions.

MPM is a very rare disease, especially in women where markers used to diagnose MPM may be also elevated in cases with cancer of the ovaries [33]. Our prediction for female candidates with tissue samples waiting for histopathological diagnostic workup resulted in 3\% false-positive decisions and 75\% true-positive decisions for a probability of $30 \%$ for mesothelin alone. A combination with calretinin did not improve the performance of mesothelin. However, our study is of limited statistical power to estimate sound results for women, based on 12 cases only. Another, more general, limitation is the case-control design, where we cannot estimate the effect of age and gender as risk factors of developing an MPM due to the matching procedure.

Despite that a large number of candidate markers has been reported for MPM in the literature, mesothelin is still the best blood-based tumor marker available and the only one with FDA approval. It has a good performance to detect MPM with AUCs ranging between 0.72 and 0.93 [34]. Our results for mesothelin are in line with similar findings that MPM patients exhibit higher mesothelin concentrations in blood than healthy controls.

Calretinin is one of the best immunohistochemical markers for MPM diagnosis [35]. This is the first study determining calretinin in plasma samples from Central America. Median calretinin concentrations in cases and controls were similar to those reported by Raiko et al. in samples from France and Germany and recently by Johnen et al. in samples from Australia and Germany $[5,11]$.

A few conditions may result in elevated marker concentrations and can thus lead to false-positive decisions. The association of pre-analytical variations with mesothelin were analyzed in several studies [36-38]. Particularly renal failure was shown to have a strong effect on the mesothelin concentrations, resulting in false-positive tests [36]. Within the framework of the MoMar study, we analyzed such pre-analytical variations in archived plasma samples from a prospective cohort of elderly subjects without malignant diseases [39]. Knowledge of possible influencing factors could improve the performance of the markers.

It is common opinion that a panel of tumor markers may improve the performance of a single marker [31, 40, 41]. However, many markers may change in parallel during carcinogenesis as could be shown in a statistical analysis of various markers applied to lung tissue [42]. Depending on the chosen probability, the inclusion of calretinin into the prediction model with mesothelin led to an improvement, albeit moderate, of the marker performance. A major reason is the tight correlation between the plasma concentrations of the two proteins in cases. However, more sophisticated decision algorithms are needed to take advantage of the combination of both markers. A more promising approach to benefit from a panel may be to combine markers from different molecular levels [31, 41]. We are currently investigating combinations of proteins with epigenetic as well as RNA markers. Initial results with mesothelin and the microRNA miR-103-3p look promising [43].

Many new MPM markers are emerging from small cross-sectional comparisons of cases and controls. Such a design suffers from methodological shortcomings [44]. The most critical problem for application in symptom-free cohorts for the early detection of MPM is a potential overestimation of the sensitivity in cases with late-stage cancer. However, this is of minor concern in our current analysis. We took advantage of marker concentrations from patients with symptoms to predict an MPM diagnosis in order to accelerate the histopathological workup, facing limited resources in Mexico. In the future, however, it would also be desirable to provide markers to be used in surveillance programs for high-risk groups of asbestos-exposed workers to allow early detection of MPM. A timely treatment at early stages of tumor development should improve the prospects of survival. Before these markers can be used in symptom-free subjects, however, their performance to detect MPM earlier has to be validated using a prospective study design [41, 45]. Because MPM is a rare disease, a sufficiently large cohort will be necessary $[32,46]$. Our study was conducted within the framework of MoMar, which is an international initiative to identify and validate markers for MPM. Within MoMar, an at-risk cohort of more than 2,700 former asbestos-exposed workers will be available for marker validation. Mesothelin and calretinin appear to be promising candidates for such a validation study. For translation into practice, marker assays not only need to have a good performance but also to be 
cost-effective, robust, and easy to apply. Because simple and robust ELISA-based assays are already available for the two markers, these requirements are basically met.

MPM represents a public health problem in Mexico. So far, the Mexican government has not followed WHO's recommendation to eliminate the use of asbestos. Since 1960, more than half a million tons had been imported from Canada, Russia, and Brazil [22]. We estimated about 5,500 deaths from MPM in Mexico 1979 to 2015, taking into account an underreporting on death certificates [47]. Just $10 \%$ of these cases were granted a general disability pension, but much fewer (ten cases) were recognized as an occupational disease in this period [20]. Nearly all cases in this study had asbestos exposure but none was recognized as an occupational disease. It is important to receive a timely diagnosis, also for granting a compensation or pension. This is in contrast to Germany, where a large fraction of incident MPM cases with former exposure to asbestos have been recognized as occupational disease [48]. Whereas the German industries provide the budget for surveillance, health care, and compensations offered by the German Social Accident Insurance, health care and pensions costs in Mexico are provided by IMSS and not by the companies causing health effects due to exposure to asbestos. A similar situation holds for INER, where patients are not insured [49].

\section{Conclusions}

This is the first study of plasma markers for the prediction of MPM in a Mexican population with a high prevalence of exposure to asbestos to accelerate the diagnostic workup of the tissue for subjects with a high probability of such a diagnosis. It is urgent for the Mexican government to upgrade the resources in molecular pathology and to ban the use of asbestos. We developed a prediction model based on the plasma concentrations of mesothelin and calretinin. Both markers combined showed a good performance to estimate an individual's probability of an MPM diagnosis in conjunction with high TPRs and acceptable FPRs.

\section{Abbreviations}

AUC: area under the curve; CI: confidence interval; ELISA: enzyme-linked immunosorbent assay; FPR: false positive rate; IQR: interquartile range; LOD: limit of detection; MPM: malignant pleural mesothelioma; OR: odds ratio; ROC: receiver operating characteristic; TPR: true positive rate.

\section{Acknowledgements}

To all patients with MPM who died from a preventable disease, and to all controls that kindly participated in the study. Thanks to all fieldwork and laboratory staff for their dedication and commitment. Thanks to Andrea Domínguez and Vanessa Sánchez who obtained their master's degree with this project. We also thank Rodrigo Gopar-Nieto, Maricruz Aceves, and Laura Alejandra Trejo Lerma, who obtained a bachelor's degree in medicine from the social service, for their contribution to interpretation of CT thorax scans and patient recruitment. We acknowledge support by the Open Access Publication Funds of the Ruhr-University Bochum. The study was funded by IMSS (Fondo de Investigación e Salud, grant FIS-IMSS-G11-935), Consejo Nacional de Ciencia y Tecnología (CONACYT) (Fondo Sectorial en Salud, grant 2012-01-181492), Bundesministerium für Bildung und Forschung (BMBF) (grant 01DN15002), and CONACYT-BMBF (Fondo de Cooperación Internacional (México-Alemania), grant 207978).

\section{Competing Interests}

The authors have declared that no competing interest exists.

\section{References}

1. Frank AL, Joshi TK. The global spread of asbestos. Annals of global health. 2014; 80: 257-62.

2. Pesch B, Taeger D, Johnen G, et al. Cancer mortality in a surveillance cohort of German males formerly exposed to asbestos. Int J Hyg Environ Health. 2010; 213: 44-51.

3. Marsili D, Comba P, Pasetto R, et al. International scientific cooperation on asbestos-related disease prevention in Latin America. Annals of global health. 2014; 80: 247-50.

4. Weber DG, Gawrych K, Casjens S, et al. Circulating miR-132-3p as a Candidate Diagnostic Biomarker for Malignant Mesothelioma. Dis Markers. 2017; 2017: 9280170.

5. Johnen G, Gawrych K, Raiko I, et al. Calretinin as a blood-based biomarker for mesothelioma. BMC Cancer. 2017; 17: 386.

6. Fuhrer G, Lazarus AA. Mesothelioma. Dis Mon. 2011; 57: 40-54.

7. Ray M, Kindler HL. Malignant pleural mesothelioma: an update on biomarkers and treatment. Chest. 2009; 136: 888-96.

8. Robinson BM. Malignant pleural mesothelioma: an epidemiological perspective. Annals of cardiothoracic surgery. 2012; 1: 491-6.

9. Hassan R, Bera T, Pastan I. Mesothelin: a new target for immunotherapy. Clin Cancer Res. 2004; 10: 3937-42.

10. Robinson BW, Creaney J, Lake R, et al. Mesothelin-family proteins and diagnosis of mesothelioma. Lancet. 2003; 362: 1612-6.

11. Raiko I, Sander I, Weber DG, et al. Development of an enzyme-linked immunosorbent assay for the detection of human calretinin in plasma and serum of mesothelioma patients. BMC Cancer. 2010; 10: 242

12. Comin CE, Novelli L, Boddi V, et al. Calretinin, thrombomodulin, CEA, and CD15: a useful combination of immunohistochemical markers for differentiating pleural epithelial mesothelioma from peripheral pulmonary adenocarcinoma. Hum Pathol. 2001; 32: 529-36.

13. Blum W, Pecze L, Felley-Bosco E, et al. Overexpression or absence of calretinin in mouse primary mesothelial cells inversely affects proliferation and cell migration. Respir Res. 2015; 16: 153

14. Hassan R, Kindler HL, Jahan T, et al. Phase II clinical trial of amatuximab, a chimeric antimesothelin antibody with pemetrexed and cisplatin in advanced unresectable pleural mesothelioma. Clin Cancer Res. 2014; 20: 5927-36.

15. Horowitz NA, Blevins EA, Miller WM, et al. Thrombomodulin is a determinant of metastasis through a mechanism linked to the thrombin binding domain but not the lectin-like domain. Blood. 2011; 118: 2889-95.

16. Goss PE, Lee BL, Badovinac-Crnjevic T, et al. Planning cancer control in Latin America and the Caribbean. Lancet Oncol. 2013; 14: 391-436. 
17. Strasser-Weippl K, Chavarri-Guerra Y, Villarreal-Garza C, et al. Progress and remaining challenges for cancer control in Latin America and the Caribbean. Lancet Oncol. 2015; 16: 1405-38.

18. Ordonez NG. Immunohistochemical diagnosis of epithelioid mesothelioma: an update. Arch Pathol Lab Med. 2005; 129: 1407-14.

19. Galateau-Salle F, Churg A, Roggli V, et al. The 2015 World Health Organization Classification of Tumors of the Pleura: Advances since the 2004 Classification. J Thorac Oncol. 2016; 11: 142-54.

20. Sistema Nacional de Derechohabientes, SINDO 2015. Sistema de Informaticon de Trabajadores Pensionados de Cesantía 2015. http://www.imss.gob.mx/ conoce-al-imss/memoria-estadistica.

21. Godleski JJ. Role of asbestos in etiology of malignant pleural mesothelioma. Thorac Surg Clin. 2004; 14: 479-87.

22. Secretaria de Economia. Plataforma Nacional de Transparencia. Instituto Nacional de Acceso a la Información: Instituto Nacional de Acceso a la Información. http://www.infomex.org.mx/gobiernofederal/home.action.

23. Agudo A, Gonzalez CA, Bleda MJ, et al. Occupation and risk of malignant pleural mesothelioma: A case-control study in Spain. Am J Ind Med. 2000; 37: 159-68.

24. Aguilar-Madrid G, Juarez-Perez CA, Markowitz S, et al. Globalization and the transfer of hazardous industry: asbestos in Mexico, 1979-2000. Int J Occup Environ Health. 2003; 9: 272-9.

25. Albin M, Jakobsson K, Attewell R, et al. Mortality and cancer morbidity in cohorts of asbestos cement workers and referents. Br J Ind Med. 1990; 47: $602-10$.

26. Burdorf A, Heederik D. Applying quality criteria to exposure in asbestos epidemiology increases the estimated risk. Ann Occup Hyg. 2011; 55: 565-8.

27. Lacourt A, Gramond C, Rolland P, et al. Occupational and non-occupational attributable risk of asbestos exposure for malignant pleural mesothelioma. Thorax. 2014; 69: 532-9.

28. Musti $\mathrm{M}$, Pollice $\mathrm{A}$, Cavone $\mathrm{D}$, et al The relationship between malignant mesothelioma and an asbestos cement plant environmental risk: a spatial case-control study in the city of Bari (Italy). Int Arch Occup Environ Health. 2009; 82: 489-97.

29. Peto $R$, Peto J. Asymptotically Efficient Rank Invariant Test Procedures. Journal of the Royal Statistical Society Series A. 1972; 135: 185-207.

30. Prentice RL. Linear rank tests with right censored data. Biometrika. 1978; 65: 167-79.

31. Tomasetti M, Santarelli L. Biomarkers for early detection of malignant mesothelioma: diagnostic and therapeutic application. Cancers (Basel). 2010; 2: $523-48$

32. Creaney J, Olsen NJ, Brims F, et al. Serum mesothelin for early detection of asbestos-induced cancer malignant mesothelioma. Cancer Epidemiol Biomarkers Prev. 2010; 19: 2238-46.

33. Davidson B. New diagnostic and molecular characteristics of malignant mesothelioma. Ultrastruct Pathol. 2008; 32: 227-40.

34. Hollevoet K, Reitsma JB, Creaney J, et al. Serum mesothelin for diagnosing malignant pleural mesothelioma: an individual patient data meta-analysis. J Clin Oncol. 2012; 30: 1541-9.

35. Ordonez NG. Value of calretinin immunostaining in diagnostic pathology: a review and update. Appl Immunohistochem Mol Morphol. 2014; 22: 401-15.

36. Hollevoet K, Bernard D, De Geeter F, et al. Glomerular filtration rate is a confounder for the measurement of soluble mesothelin in serum. Clin Chem. 2009; 55: 1431-3.

37. Weber DG, Johnen $G$, Taeger $D$, et al. Assessment of Confounding Factors Affecting the Tumor Markers SMRP, CA125, and CYFRA21-1 in Serum. Biomark Insights. 2010; 5: 1-8

38. Park EK, Thomas PS, Creaney J, et al. Factors affecting soluble mesothelin related protein levels in an asbestos-exposed population. Clin Chem Lab Med. 2010; 48: 869-74.

39. Casjens S, Weber DG, Johnen G, et al. Assessment of potential predictors of calretinin and mesothelin to improve the diagnostic performance to detect malignant mesothelioma: results from a population-based cohort study. BMJ open. 2017; 7: e017104.

40. Pesch $B$, Taeger $D$, Johnen $G$, et al. Screening for bladder cancer with urinary tumor markers in chemical workers with exposure to aromatic amines. Int Arch Occup Environ Health. 2014; 87: 715-24

41. Pesch $B$, Brüning $T$, Johnen $G$, et al. Biomarker research with prospective study designs for the early detection of cancer. Biochim Biophys Acta. 2014; 1844: 874-83.

42. Pesch B, Casjens S, Stricker I, et al. NOTCH1, HIF1A and other cancer-related proteins in lung tissue from uranium miners--variation by occupational exposure and subtype of lung cancer. PLoS One. 2012; 7: e45305.

43. Weber DG, Casjens S, Johnen G, et al. Combination of MiR-103a-3p and mesothelin improves the biomarker performance of malignant mesothelioma diagnosis. PLoS One. 2014; 9: e114483.

44. Behrens T, Bonberg N, Casjens S, et al. A practical guide to epidemiological practice and standards in the identification and validation of diagnostic markers using a bladder cancer example. Biochim Biophys Acta. 2014; 1844: $145-55$

45. Panou V, Vyberg M, Weinreich UM, et al. The established and future biomarkers of malignant pleural mesothelioma. Cancer Treat Rev. 2015; 41: 486-95.
46. Roe OD, Creaney J, Lundgren S, et al. Mesothelin-related predictive and prognostic factors in malignant mesothelioma: a nested case-control study. Lung Cancer. 2008; 61: 235-43.

47. Aguilar-Madrid G, Robles-Perez E, Juarez-Perez CA, et al. Case-control study of pleural mesothelioma in workers with social security in Mexico. Am J Ind Med. 2010; 53: 241-51.

48. Hagemeyer $\mathrm{O}$, Otten $\mathrm{H}$, Kraus T. Asbestos consumption, asbestos exposure and asbestos-related occupational diseases in Germany. Int Arch Occup Environ Health. 2006; 79: 613-20.

49. Frenk Julio. Acuerdo por el que la Secretaria de Salud da a conocer las reglas de operacion e indicadores de gestion y evaluacion del Seguro Popular: Diario Oficial, México, 2002. http://www.salud.gob.mx/unidades/cdi/nom/ compi/assrosps.html. 